\title{
Genèse du nouveau master « Histoire des sciences et techniques, humanités numériques et médiations culturelles $»$ : de l'articulation entre la recherche et la formation au Centre François Viète de l'Université de Bretagne Occidentale
}

\author{
Gregory Chambon et Sylvain Laubé \\ Centre F. Viète (EA 1161), Université Européenne de Bretagne, Université de Brest, France
}

\begin{abstract}
Résumé. L'objet de cet article est de montrer comment la dynamique de création simultanée, à Brest et depuis 2008, d'une nouvelle équipe de recherche et d'un nouveau master recherche à distance en 2012 s'est traduit par une proposition originale de formation par la recherche. Nous détaillons les spécificités de cette dernière. D'une part, elle prend fortement appui sur des axes de recherches et des nouvelles méthodologies en histoire des sciences et des techniques, qui s'inspirent des travaux sur la culture matérielle et la compréhension des systèmes complexes. En outre, nous montrons que des questions de recherche et de formation y sont intégrées concernant le développement ou l'usage des outils numériques (web sémantique, réalité virtuelle ou augmentée, etc.) pour la recherche en SHS et médiations culturelles. L'équipe de Brest s'inscrit de ce fait dans le champ des travaux de recherches en humanités numériques en très forte collaboration avec notamment des laboratoires d'informatique. Au travers des thématiques traitées, des méthodologies enseignées, d'une pédagogie universitaire basée sur une classe virtuelle ainsi que de la nature des différents partenaires qui y enseignent, nous montrons ainsi que le master en question constituent un laboratoire où il est possible d'expérimenter de nouvelles pratiques de formation par la recherche.
\end{abstract}

\section{Introduction}

L'objet de cet article est de présenter la synthèse de deux communications présentées en $2013^{1}$. Il s'agit ici de montrer comment la dynamique (opérée depuis 2008) de création à la fois d'une nouvelle équipe de recherche à Brest en tant que qu'antenne du Centre F. Viète (EA 1161) de l'Université de Nantes et d'un nouveau master recherche à distance en 2012 s'est traduit par une proposition de formation par la recherche qui prend fortement appui sur des axes de recherches et des nouvelles méthodologies en histoire des sciences et des techniques. Ces méthodes s'inspirent des travaux sur la culture matérielle et

\footnotetext{
${ }^{1}$ G. Chambon \& S. Laubé (2013). Outils pour la recherche, outils pour les enseignements de master : "culture matérielle », " approche systémique » et usage des TIC ; Colloque "SHST2013-UPEC : Sciences Humaines en Sciences et Techniques. Les sciences humaines dans les parcours scientifiques et techniques professionnalisant : quelles finalités et quelles modalités pratiques ? 7-8 févr. 2013 Créteil (France); http://shst2013-upec.sciencesconf.org/10680 S. Laubé (2013). Formation des étudiants à l'Université et corpus en ligne. Journée d'étude « Usages des sources numériques en histoire des sciences et des techniques III », 29 nov. 2013, Cité des Sciences et de l'Industrie, Paris ; http://www.koyre.cnrs.fr/IMG/pdf/crhst_progr.29_11_13_ok_.pdf.
}

This is an Open Access article distributed under the terms of the Creative Commons Attribution License 4.0, which permits unrestricted use, distribution, and reproduction in any medium, provided the original work is properly cited. 
la compréhension des systèmes complexes. Par ailleurs, des questions de recherche et de formation y sont intégrées concernant le développement ou l'usage des outils numériques (web sémantique, réalité virtuelle ou augmentée, etc.) pour la recherche en SHS et médiations culturelles. L'équipe de Brest s'inscrit de ce fait dans le champ des travaux de recherches en humanités numériques en très forte collaboration avec notamment des laboratoires d'informatique. Au travers des thématiques traitées, des méthodologies enseignées, d'une pédagogie universitaire basée sur une classe virtuelle ainsi que de la nature des différents partenaires qui y enseignent, le master recherche " Histoire des sciences et des techniques, humanités numériques et médiations culturelles » constituent un laboratoire où il est possible d'expérimenter de nouvelles pratiques de formation par la recherche.

\section{Contextes}

\subsection{La politique de recherche de l'équipe sous-jacente au master}

Le site du Centre F. Viète (EA 1611) de l'Université de Bretagne Occidentale a été créé le $1^{\mathrm{er}}$ janvier 2012. Les thématiques qui structurent la recherche sur ce site s'articulent entre deux pôles (STeMA et $\mathrm{STeMoC})^{2}$ et incluent des questions de recherches relatives aux humanités numériques et à la médiation culturelle envers des publics scolaires et périscolaires ${ }^{3}$. Elles s'inscrivent en forte collaboration avec un groupe d'informaticiens de Telecom-Bretagne ${ }^{4}$, par ailleurs chercheurs associés au laboratoire, ainsi qu'avec Océanopolis, centre de culture scientifique spécialisé dans le domaine de la mer ${ }^{5}$. La dimension « médiations culturelles » s'est déjà traduite par la publication de plusieurs ouvrages destinés aux chercheurs et aux enseignants ${ }^{6}$. La dimension « humanités numériques » pour l'histoire des sciences et des techniques s'inscrit par ailleurs dans le cadre du groupe de recherche «SemanticHPST » avec une collaboration avec les Universités de Lorraine et de Montpellier ${ }^{7}$. Les travaux de recherche de ce groupe porte sur des questions de modélisation de connaissances et de développement d'outils pour la recherche en SHS basé sur le web sémantique. La politique menée dans l'équipe cherche à fédérer les chercheurs sur des questions transversales permettant de faire dialoguer à la fois les périodes historiques (mondes antiques, modernes et contemporains), les thématiques (comme par exemple, les sciences et technologies de la mer et des fleuves). Elle s'appuie par ailleurs sur une réflexion épistémologique concernant les méthodologies d'une histoire des sciences et des techniques tenant de la culture matérielle, de l'approche systémique et des humanités numériques. Nous reviendrons sur ce point plus loin dans le texte.

Adossé au Centre F. Viète de l'UBO et aux méthodes et thématiques de recherche qui y sont développées le dossier de création du master recherche «Histoire des sciences et techniques, humanités numériques et médiations culturelles ${ }^{8}$ (master HST), a été déposé en novembre 2011 aux instances de l'Université de Bretagne Occidentale. Le titre même du master résume nos principales thématiques de recherche, développées plus haut : des recherches en histoire des sciences et techniques, en humanités numériques et en médiation dans le domaine du patrimoine scientifique et technique. En travaillant sur des questions de recherche en histoire des sciences et techniques et Humanités numériques, il

\footnotetext{
${ }^{2}$ STeMA : Sciences et Techniques des Mondes Antiques; STeMoC : Sciences et Techniques des Mondes Modernes et Contemporains.

${ }^{3}$ Concernant cette dernière thématique, l'équipe de Brest avait participé à la fondation du groupe ReForEHST qui avait provoqué plusieurs journées d'études en lien avec la formation des maîtres et co-organisé plusieurs sessions dans les congrès de la société européenne d'histoire des sciences (European Society for History of Science, ESHS).

${ }^{4}$ Dans le cadre du LabSTICC et dirigé par S. Garlatti.

5 http://www . oceanopolis.com/ (consulté 9.10.14).

6 Voir (Ferrière 2011; Bernard et al. 2010; Bruneau et al. 2012).

7 Voir http://www.msh-lorraine.fr/index.php?id=671 et http://semhpst.hypotheses.org/ (consultés 9.10.14)

8 Voir la présentation du master (M1 et M2) à Brest ici : http://formations.univ-brest.fr/fiche/FR_RNE_0290346U_ PROG20224/FR_RNE_0290346U_PROG20230/presentation (consulté 9.10.14).
} 
vise à former des chercheurs et des professionnels de la médiation pour participer à des projets pluridisciplinaires, afin de valoriser le patrimoine et la culture scientifique et techniques. Ce point sera développé plus loin (§4).

\subsection{Le contexte institutionnel de I'UBO}

Outre la politique de recherche de l'équipe, le contexte institutionnel a joué un rôle essentiel dans la genèse du master, et les choix entrepris ont essayé de répondre aux attentes des instances. Le travail effectué sur sa conception avait en effet, dans un premier temps, porté sur un master 2, qui devait être le pendant brestois du master 2 «Histoire des sciences et des techniques » de l'Université de Nantes ${ }^{9}$. Les entretiens des instances de l'UBO avec la Direction Générale pour l'Enseignement Supérieur et l'Insertion Professionnelle ont rapidement fait apparaître la nécessité de créer également une année de master 1. En effet, comme le master était proposé par l'IUFM ${ }^{10}$ (qui était alors notre composante de rattachement à l'UBO), il a été inscrit dans la mention « Métier de l'éducation et de la formation ». En effet, une formation sur deux ans permettait de renforcer la spécialité « Histoire des sciences et techniques, TIC et médiations culturelles », créée spécialement pour le master, et de toucher directement un public de troisième année de licence.

Plusieurs heures de cette première année de master 1 ont alors été mutualisées avec le master Professorat des Ecoles (ou master PE dans la suite) de l'IUFM, afin d'essayer de répondre à la question de l'hétérogénéité et de la « mise à niveau » du public visé, issu de parcours littéraires ou scientifiques. Nous avons donc décidé de faire suivre à nos étudiants les enseignements de mathématiques, de physique et des sciences de la vie et de la Terre, proposé dans le master PE. Ils nous paraissaient bien adaptés à nos attentes, puisque, d'une part, ils correspondaient à un niveau scientifique accessible (c'est-à-dire le niveau collège) et, d'autre part, ils étaient destinés à préparer à l'enseignement des sciences, une perspective compatible avec la formation à la médiation culturelle proposée dans le master, en particulier à destination des publics scolaires.

Après sa validation par le ministère et les instances de l'UBO, le master est entré dans l'offre de formation de l'UBO pour la rentrée de septembre 2012. Les effectifs pour une première année d'un master recherche ont été plus qu'honorables ; 5 étudiants inscrits en M1 et 12 en M2 (dont le public était composé uniquement d'enseignants du secondaire $)^{11}$. Les professeurs de collège et de lycée ont continué à représenter la majorité des étudiants pour l'année suivante 2013-2014. La possibilité de suivre une partie ou l'ensemble des cours en classe virtuelle ${ }^{12}$ a largement contribué à attirer ce type de public, avec des horaires professionnels variables.

\subsection{Influence des réformes de 2012-13 sur l'évolution du master}

Depuis la fin de l'année 2012, des réformes ont touché l'enseignement supérieur et mis fin aux IUFM au profit des nouvelles Ecoles Supérieures du Professorat et de l'Education (ESPE). Les profondes modifications opérées sur les maquettes des masters « enseignements » ont donc directement influencé le contenu et la structure des unités d'enseignement de ce master ; en particulier, les mutualisations envisagées décrites plus haut n'étaient plus possibles. De plus, le processus de création de l'ESPE de Bretagne, destinée à rejoindre le PRES de la région Bretagne (l'Université Européenne de Bretagne), nous a amenés à revoir le cadre administratif d'accueil du master et à l'intégrer en septembre 2014 dans l'offre de formation de l'UFR Lettres et Sciences Humaines de l'UBO.

\footnotetext{
${ }^{9}$ Voir la présentation du master (M2) à Nantes ici : http://www.univ-nantes.fr/SI00207/0/fiche___formation/ (consulté 9.10.14).

${ }^{10}$ Institut Universitaire de Formation des Maîtres.

${ }^{11}$ Les étudiants inscrits en master 1 provenaient d'une troisième année de licence littéraire ou scientifique.

${ }^{12}$ Le système utilisé est un service fourni par l'Université Européenne de Bretagne : http: //e-learning. ueb.eu (consulté le 09.10.14).
} 
Le choix de cette composante reposait en grande partie sur le type de public visé par le master, qui ne concerne pas uniquement le monde enseignant. Nous visons en réalité quatre types de public : $1^{\circ}$ ) Des étudiants issus de filières littéraires ou scientifiques (en formation initiale) $2^{\circ}$ ) des étudiants en formation continue avec notamment des professionnels de la médiation en sciences et techniques ainsi que des professionnels du patrimoine scientifique et technique c) des enseignants. La seconde raison principale de ce choix nous ramène à la question de l'articulation à la recherche, puisqu'elle concerne l'adossement du master au Centre François Viète, hébergé lui-même à l'Institut Brestois des Sciences Humaines et Sociales dans l'UFR Lettres et Sciences Humaines. Les thèmes des mémoires de nos étudiants sont en effet directement liés à des projets de recherche en cours dans le CFV, sur des questions d'Humanités numériques, d'Histoire des sciences et techniques et de médiations culturelles. Cette articulation très serrée entre recherche et formation ne peut prendre son sens que dans le cadre d'un rattachement à l'université et dans une Unité de Formation et de Recherche (UFR).

L'ensemble de ces changements nous a conduits à transformer en particulier l'année de master 1 . Si nous avons décidé de maintenir une unité d'enseignement sur les savoirs en sciences, technologies et mathématiques à la place de la précédente auparavant mutualisée avec le master PE, nous avons également souhaité d'en faire apparaître deux nouvelles correspondant aux spécificités du master en adéquation avec le type de public visé : la première intitulée «Humanités numériques et enseignement » et la seconde « Médiation et patrimoine scientifique : des contenus HST à la mise en œuvre d'un projet » qui se déroule sur deux semestres.

Aussi, le programme proposé aux étudiants se présente actuellement comme suit : ${ }^{13}$

\begin{tabular}{|c|c|c|}
\hline 莺 & \multicolumn{2}{|c|}{$\begin{array}{l}\text { Sciences et société (s) I : textes de référence en HST (36h) } \\
\text { Savoirs en Sciences, Technologies et Mathématiques I (75h) } \\
\text { Humanités numériques et enseignement ( } 36 \mathrm{~h}) \\
\text { Initiation à la recherche I ( } 36 \mathrm{~h}) \\
\text { - Médiation et patrimoine scientifique I : des contenus HST à la mise en oeuvre d'un projet (36h) } \\
\text { Anglais pour la recherche I ( } 22 \mathrm{~h})\end{array}$} \\
\hline 岕 & \multicolumn{2}{|c|}{$\begin{array}{l}\text { Sciences et société(s) II : textes de référence en HST (72h) } \\
\text { Savoirs en STM II (66h) } \\
\text { Stage d'observation (12h) } \\
\text { Initiation à la recherche II ( } 36 \mathrm{~h}) \\
\text { - Médiation et patrimoine scientifique II : des contenus HST à la mise en oeuvre d'un projet (24h) } \\
\text { Initiation aux langues de la méditerranée antique pour l'étude et la valorisation des textes anciens } \\
\text { (Grec, Latin, Akkadien) (48h) }\end{array}$} \\
\hline \multirow[b]{2}{*}{ 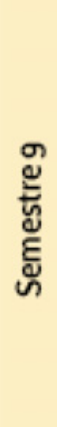 } & \multicolumn{2}{|c|}{$\begin{array}{l}\text { Histoire de la transmission et de la diffusion des savoirs scientifiques et techniques ( } 30 \mathrm{~h}) \\
\text { Méthodes et outils de la recherche pour l'HST, les humanités numériques et la médiation ( } 60 \mathrm{~h}) \\
\text { Anglais pour la recherche II ( } 24 \mathrm{~h})\end{array}$} \\
\hline & $\begin{array}{l}\text { Parcours STeMA } \\
\text { (Sciences et Techniques des Mondes Antiques) } \\
\text { Sources et méthodologie (24h) } \\
\text { HST, patrimoine et médiations culturelles '24h) }\end{array}$ & $\begin{array}{l}\text { Parcours STeMOC } \\
\text { (Sciences et Techniques des Mondes Modernes } \\
\text { et Contemporains) } \\
\text { Sources et méthodologie }(24 \mathrm{~h}) \\
\text { Option (1 au choix) : (24h) } \\
\text { - Histoire des mathématiques } \\
\text { - Histoire des sciences de la vie et de la terre } \\
\text { - Histoire de la physique et de la chimie }\end{array}$ \\
\hline
\end{tabular}

13 Voir le détail du programme et des informations sur le master à : http://formations.univ-brest.fr/fiche/FR_RNE_ 0290346U_PROG20224/FR_RNE_0290346U_PROG20230/presentation 
Les sciences humaines dans les parcours scientifiques et techniques professionnalisants

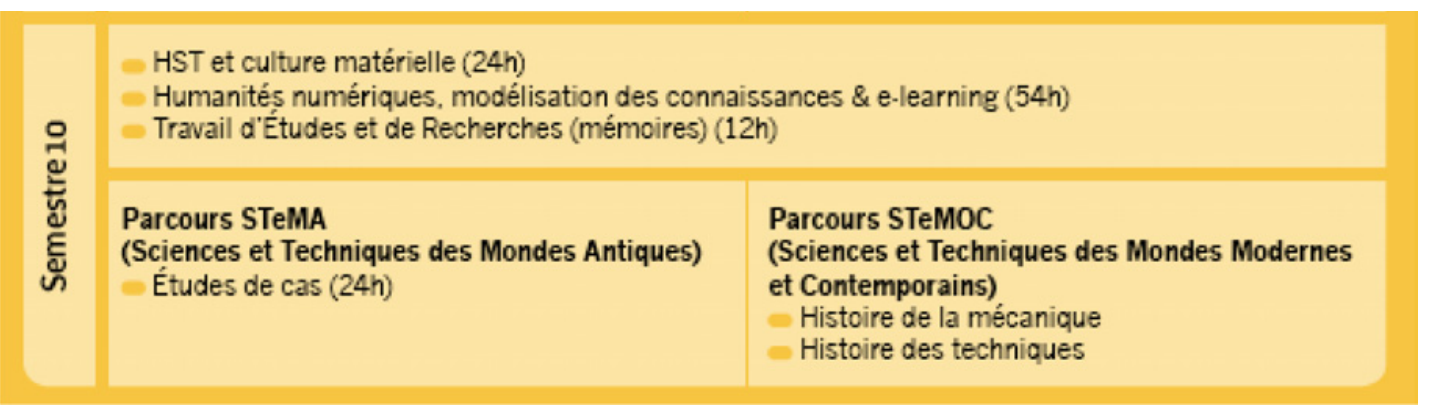

\subsection{Conclusion : une double préoccupation pour la formation et la recherche}

En élaborant l'organisation et le contenu de ces enseignements, nous ne nous sommes pas seulement posé des questions sur l'adossement à la recherche, mais aussi d'emblée celle de l'utilité sociale et professionnelle du master. En effet, s'il s'agit d'un master recherche qui a pour premier débouché la préparation d'une thèse dans le domaine des SHS ou des STIC ${ }^{14}$, l'insertion professionnelle de nos étudiants nous a paru essentielle, qu'ils continuent en thèse ou s'inscrivent immédiatement après l'obtention de leur dans une recherche d'emploi ou encore dans une évolution de leur carrière (lorsqu'ils ont déjà des activités professionnelles). Cet aspect se traduit par deux points essentiels à nos yeux : tout d'abord, une approche du terrain d'exercice professionnel et des problèmes de recherche posés par les divers acteurs : médiation culturelle/Océanopolis, Archives Municipales, Conservatoire Botanique, DRAC, Musée, etc.) qui engendrent des propositions de sujet de mémoire de recherche avec ces mêmes acteurs. Les objectifs sont expressément que les étudiants aient des compétences dans la gestion de projet pluridisciplinaire et inter professionnel. Après cette présentation des différents contextes, abordons le vif du sujet.

\section{Les méthodologies de travail et de recherche du CFV à l'UBO}

\subsection{Méthodologie de l'histoire}

Les recherches entreprises par les membres de l'équipe CFV du pôle brestois s'organisent autour de trois axes principaux : la notion de «culture matérielle », l'approche systémique des artefacts et les humanités numériques.

La notion de « culture matérielle », héritée des études pionnières de l'anthropologie, initiées plus particulièrement dans le monde anglo-saxon (material culture) ${ }^{15}$, a été reprise par plusieurs disciplines, comme l'archéologie, l'ethnographie, l'histoire, la muséologie. Son caractère polysémique a donné lieu à diverses interprétations selon les disciplines. Si l'on caractérise (grossièrement) une culture par un ensemble de traits caractéristiques qui la distinguent d'une autre culture ${ }^{16}$, la culture matérielle est le plus souvent comprise comme la part de "matérialité » dans cet ensemble et renverrait donc aux traits culturels visibles et tangibles : les objets, les outils, et peut-être, au-delà, les gestes et les techniques associés à leurs usages. Il y a là une ambiguïté entre les notions de «culture matérielle » et «patrimoine matériel »; c'est en particulier sur des critères de tangibilité et de visibilité que l'UNESCO fonde sa distinction entre le « patrimoine matériel », compris comme l'ensemble des

\footnotetext{
${ }^{14}$ La première de master s'est traduit par l'inscription en thèse de deux étudiants en co-direction STIC et SHS, l'une se déclinant dans l'Ecole Doctorale SHS, l'autre dans l'Ecole Doctorale SICMA (i.e. STIC).

15 Voir Lewis H. Morgan, La Société archä̈que, 1877, qui a fondé une classification des sociétés sur des critères matériels. Auguste Henry Lane-Fox Pitts Rivers n'emploie pas le terme dans son On the evolution of culture de 1875, mais s'intéresse à l'étude des material arts.

16 Pour une définition plus fine, voir (Rocher 1992, p. 101-127).
} 
monuments, constructions, biens mobiliers, collections d'objets, outils et équipements, et le « patrimoine immatériel », qui regrouperait : ${ }^{17}$

«...les traditions ou les expressions vivantes héritées de nos ancêtres et transmises à nos descendants, comme les traditions orales, les arts du spectacle, les pratiques sociales, rituels et événements festifs, les connaissances et pratiques concernant la nature et l'univers ou les connaissances et le savoir-faire nécessaires à l'artisanat traditionnel. »

Dans les premières études anthropologiques du XIXe siècle, la culture matérielle était d'ailleurs distinguée de la culture spirituelle, associée au folklore. Depuis cette anthropologie des techniques, qui cherchait à reconstituer l'histoire, l'usage et le rôle symbolique des objets techniques, la recherche s'est orientée progressivement vers la dimension sociale et humaine des techniques, qui ne se limite pas forcément aux objets. P. Lemonnier, après avoir précisé que « ... tout ce qui concerne l'action de l'homme sur la matière relève de la technique » (Lemonnier 2010, p. 50), défend par exemple dans son projet de «technologie culturelle », un paradigme de trois ordres : les objets compris comme des moyens d'action sur la matière, les processus mis en œuvre dans l'usage et la conception de ces objets et les connaissances associées à ces usages. La notion de « culture matérielle » devient là équivalente à celle de « phénomènes techniques » au sein d'un groupe social déterminé.

Selon la discipline, l'aspect « matérialité » est donc plus ou moins interrogée, ainsi que l'interaction étroite entre homme et objet ; la culture matérielle, loin de se réduire aux simples objets physiques, intègre donc maintenant la relation entre les objets et les sujets (Rosselin \& Julien 2005, p. 6-7). Cette interprétation suscite depuis peu de nombreuses questions épistémologiques de nature interdisciplinaire, portant sur le mécanisme des relations entre l'Homme, sa production matérielle et ses idées, en particulier pour les périodes préhistoriques pour lesquelles la culture matérielle semble constituer le seul moyen d'accès aux sociétés du passé ; plusieurs réflexions intéressantes concernant la dimension «idéelle» de la culture matérielle ont été ainsi initiées chez plusieurs spécialistes des sciences humaines et sociales ${ }^{18}$.

"Tout objet doit être étudié : $1^{\circ}$ en lui-même; $2^{\circ}$ par rapport aux gens qui s'en servent ; $3^{\circ}$ par rapport à la totalité du système observé. Le mode de fabrication donnera lieu à une enquête approfondie » (Mauss 1967, p. 31). Le troisième point pointé par M. Mauss indique que la culture matérielle nécessite aussi d'être pensée en tant que système. Nous y intégrons des facteurs d'échelles, de complexité en posant notamment la question de : 1) de l'évolution de macro-systèmes technologiques en considérant qu'un ou des ensembles d' « artefacts » avec les communautés de pratiques et les savoirs associés constituent des éléments de ce macro-système ${ }^{19}$;2) des méthodes adéquates de compréhension des systèmes complexes du fait des facteurs d'échelles (Le Moigne 2004).

Enfin, la réflexion épistémologique sur les objets et les méthodes de l'histoire que nous souhaitons invoquer est aussi constitutive des travaux de recherches menés dans le groupe SemanticHPST sur les questions de modélisation de connaissances pour développer dans le cadre du web sémantique des ontologies de référence notamment pour renseigner de manière adéquate les jeux de métadonnées et rendre notamment automatique la composition de corpus numériques à partir de sources numériques en ligne ${ }^{20}$. En effet, ces ontologies nécessitent une explicitation forte à la fois des objets d'études, des

\footnotetext{
17 Voir : http: //www. unesco.org/culture/ich/index.php?lg=fr\&pg=00002 (consulté 9.10.14).

18 Nous renvoyons par exemple à l'un des axes de recherche du Labex Sciences Archéologiques de Bordeaux (LaScArBx) et à la journée d'étude "Au delà du caractère fonctionnel, la dimension "idéelle" de la culture matérielle ", qui a été organisée à Bordeaux le 31 mai 2013 dans le cadre de ce Labex, et qui a questionné les relations entre production matérielle et production d'idées : http://www.prehistoire.org/offres/gestion/actus_515_16767-911/au-dela-du-caracterefonctionnel-la-dimension-ideelle-de-la-culture-materielle.html (consulté 9.10.14).

19 Voir par exemple (Gille 1979; Hughes 1987).

${ }^{20}$ Nous organisons sur ce sujet une session intitulée «Web sémantique et humanités numériques en histoire, philosophie des sciences et des techniques : quelles continuités et ruptures dans les pratiques et les problématiques ? 》 dans le cadre du congrès de la Société Française d'Histoire des Sciences et Techniques à Lyon les 28, 29 et 30 avril 2014 (http://sfhst2014lyon.sciencesconf .org/).
} 
concepts à l'œuvre et des pratiques de recherches. Une thèse est en cours sur ces questions dans le cadre d'une étude comparatiste des ports de Brest et de Mar del Plata (Argentine) ${ }^{21}$.

En tant qu'historiens des sciences et des techniques, il nous a semblé intéressant d'essayer d'interpréter selon notre point de vue cette notion de culture matérielle, d'approche systémique et humanités numériques et d'apporter dans ce débat une approche issue de plusieurs études de cas, de l'Antiquité à l'Epoque Moderne ${ }^{22}$. Ces réflexions sont abordées en particulier dans le cadre du séminaire de recherche du Centre François Viète, suivi par les étudiants de master 2.

\subsection{Valorisation des recherches : médiation culturelle}

La valorisation des recherches en HST (et humanités numériques) en terme de médiation au sein du laboratoire à Brest se traduit par plusieurs types d'activités de recherche avec un rapprochement très étroit avec le LabSTICC, le Centre Européen de Réalité Virtuelle et l'Ecole Navale sur deux types de sujets de recherche avec la collaboration effective avec de partenaires acteurs de la culture scientifique. A titre d'exemple, une thèse est en cours en collaboration avec Océanopolis, le LabSTICC et le CFV sur la valorisation et la compréhension d'un espace géographique (la rade de Brest) d'un point de vue écologique (au travers d'une histoire de la coquille Saint Jacques) et anthropique (au travers d'une histoire de la construction navale $)^{23}$. La thématique de cette thèse s'inscrit à la fois en terme d'histoire des sciences et des techniques et d'EIAH (Environnement Informatique pour l'Apprentissage Humain). Par ailleurs, une autre thèse démarre en septembre 2014 dans le cadre du projet Eleppu en histoire de l'Antiquité ${ }^{24}$ en collaboration avec L'Ecole Navale intégrant des questions de modélisation 3D d'une embarcation mésopotamienne. Autre exemple symptomatique de nos activités de recherche en cours, la modélisation 3D en deux étapes de l'atelier des forges de l'arsenal de Brest (en collaboration avec Brest Métropole Océane) qui présente un fort intérêt historique et patrimonial, notamment par la présence d'un marteau-pilon Schneider de 1865 encore en activité il y a un an. A l'aide d'un LIDAR dans le cadre d'une collaboration avec le service Pôle Image de $1^{\prime} \mathrm{UBO}^{25}$, il s'agit d'enregistrer une image statique 3D de l'atelier des forges et du marteau-pilon in situ (avant modification des lieux ou déplacement de certains éléments), puis de répliquer cet environnement industriel en réalité virtuelle 3D immersive (à partir des archives et des plans) avec un projet de capture des gestes professionnels des forgerons en collaboration avec le CERV ${ }^{26}$. L'objectif étant de constituer un jeu d'archives 3D statiques et dynamiques concernant les artefacts en jeu associés aux procédures effectuées par les équipes au travail. Ce jeu d'archives 3D étant destiné au travail de l'historien et à la médiation culturelle à mettre en place en incluant le résultat de l'étude historique.

\section{Objectifs du master : formation par et pour la recherche}

L'idée de ce master est née d'un constat. À travers leurs différentes activités de recherche en histoire des sciences et techniques, de l'Antiquité à l'Epoque moderne, les membres de l'équipe sont confrontés à des questions de valorisation du patrimoine scientifique et technique, notamment à partir des corpus

\footnotetext{
21 Voir http: //brmdp.hypotheses.org/ (consulté 09.10.14).

22 Nous organisons sur ce sujet une session intitulée «Culture matérielle, Histoire des sciences et des techniques » dans le cadre du congrès de la Société Française d'Histoire des Sciences et Techniques à Lyon les 28, 29 et 30 avril 2014 (http://sfhst2014lyon.sciencesconf.org/resource/page/id/5, session 18).

23 Voir http://departements.telecom-bretagne.eu/info/theses/theses_en_cours/leborgne_patrice (consulté 9.10.14).

24 http://python.espe-bretagne.fr/blog-maeva-boudjema/?author=1 (consulté 9.10.14).

25 http://www-iuem.univ-brest.fr/fr/linstitut/services-moyens-communs/services-communs/pole-image (consulté 9.10.14).

${ }^{26}$ http: //www.cerv.fr/?page_id=51 (consulté 9.10.14).
} 
étudiés. Nous nous sommes aperçus qu'une demande réelle de spécialistes dans ce domaine se faisait sentir au sein des diverses institutions et bailleurs de fonds avec lesquelles nous travaillons (ville, région, Maisons des Sciences Humaines, Musée, DRAC), dans le cadre plus général, défini par la France et l'Europe, des projets «Sciences \& Sociétés », dont l'objectif est de diffuser la culture scientifique et technique auprès des publics scolaires et du grand public.

Le master a été créé comme réponse à cette demande, en formant des chercheurs polyvalents possédant les compétences suivantes :

$1^{\circ}$ ) Concevoir des outils innovants de médiation culturelle en histoire des sciences et techniques devant divers publics ;

$2^{\circ}$ ) Concevoir et gérer des projets Recherche \& Développement pour la médiation et l'éducation impliquant des partenaires pluridisciplinaires (musées, acteurs du patrimoine, entreprises, laboratoires de recherche) ;

$3^{\circ}$ ) Expertiser et conseiller les décideurs et bailleurs de fonds dans le domaine de la valorisation du patrimoine scientifique et technique.

$4^{\circ}$ ) Posséder des compétences historiques et méthodologiques permettant d'assurer une recherche documentaire spécialisée.

$5^{\circ}$ ) Connaître la réalité professionnelle des lieux de transmission et de diffusion des savoirs scientifiques et techniques.

L'objectif est donc de former des spécialistes qui puissent servir d'interlocuteurs à des bailleurs de fonds, en sachant réinvestir leurs diverses compétences au sein d'un projet et dialoguer avec d'autres spécialistes (professionnels des musées, spécialistes des Technologies de l'Informations et de la Communication, chercheurs en Sciences Humaines ou en Sciences dures, etc.).

Cet objectif nous a donc permis de définir les quatre types de publics visés, présentés plus haut. L'adéquation de cette formation avec le quatrième type, les enseignants, mérite d'être développée ${ }^{27}$. Outre l'aspect diplômant, les enseignants du secondaire s'inscrivant à un master d'histoire des sciences et techniques souhaitent, la plupart du temps, se créer (ou enrichir) une culture historique dans leur discipline, afin de mieux maîtriser cette dernière, l'inscrire dans des débats passés ou contemporains, et ainsi mieux développer de nouvelles stratégies dans leurs pratiques pédagogiques ${ }^{28}$. Même si nous proposons en master 2 des UE dédiées à l'histoire des disciplines ${ }^{29}$ (SVT, sciences physiques, mathématiques, mécanique et techniques), notre approche de la formation est, comme notre approche de la recherche, fortement interdisciplinaire (voir \$3). Nous travaillons sur des cas d'études en histoire des sciences et techniques, qui mobilisent plusieurs champs disciplinaires, du point de vue des méthodes et des problématiques. L'objectif, avec ce public d'enseignants, est donc également de les former à des questions de recherche concernant la diffusion de la culture scientifique et la valorisation du patrimoine, qui puissent les conduire non seulement à réinvestir leurs connaissances historiques de leur propre discipline, mais également à dialoguer avec des collègues afin de monter des projets pluridisciplinaires au niveau local ou régional ${ }^{30}$.

Le seul découpage opéré dans les UE du master n'est donc pas d'ordre disciplinaire mais d'ordre chronologique. En raison de la spécificité des périodes étudiées par les chercheurs du CFV intervenant dans le master, qui concernent l'Antiquité d'une part et les Epoques Moderne et Contemporaine d'une

\footnotetext{
${ }^{27}$ L'adéquation avec les autres publics (étudiants pour la formation initiale et professionnels de la médiation pour la formation continue) est évidente.

28 Voir à ce sujet les réflexions d'Alain Bernard, qui souligne les difficultés d'une telle démarche et en décrit tous les enjeux (Bernard 2009).

${ }^{29}$ Ces UE sont mutualisées avec le master « histoire des sciences et techniques » de Nantes.

${ }^{30}$ A titre d'exemple, voir le projet LIBROS de Céline Le Gall, professeur de Latin au Lycée Kerichen à Brest : http://liaison-lycees-ubo.univ-brest.fr/pages/libros (consulté 9.10.14). Cette enseignante a par ailleurs obtenu brillamment son diplôme de master 2 en juin 2013 et s'est engagé dans une thèse portant sur la traduction des mémoires des prix de l'Académie Royale des Sciences de Giovanni Poleni portant sur la thématique de la mer.
} 
autre, nous proposons deux parcours en masters 2: le parcours STEMA (Sciences et Techniques des Mondes Antiques) et le parcours STEMOC (Sciences et Techniques des Mondes Moderne et Contemporain).

\section{Mise en œuvre des enseignements}

La mise en œuvre des enseignements s'effectue dans le cadre de l'utilisation d'une classe virtuelle SVI mis à disposition des enseignants chercheurs par l'Université Européenne de Bretagne ${ }^{31}$. Cette classe SVI permet de publier en ligne pour l'ensemble des étudiants une bibliographie de référence dans divers formats (texte, image, vidéo, lien internet, etc.), d'enregistrer en totalité un cours (son, image, diaporama ainsi que toute activité effectuée sur l'écran par l'enseignant) et de le restituer en streaming ou en téléchargement. Un cours se déroule en mode synchrone avec deux types de publics, l'un en présentiel possédant un portable connecté en wifi sur le réseau de l'UBO, l'autre à distance. Les étudiants peuvent interagir avec l'enseignant au travers d'un micro ou d'un chat. En mode asynchrone, l'ensemble des étudiants peuvent récupérer les documents en ligne ainsi que la totalité du cours. En termes de suivi pédagogique, les étudiants ont la possibilité de communiquer par mail l'enseignant responsable de l'UE.

Un des objectifs du master est que chaque étudiant possède la culture numérique adéquate pour les usages professionnels liés à la recherche. Ce point se traduit par la mise en place d'un environnement numérique très diversifié (voir schéma ci-dessous ${ }^{32}$ ).

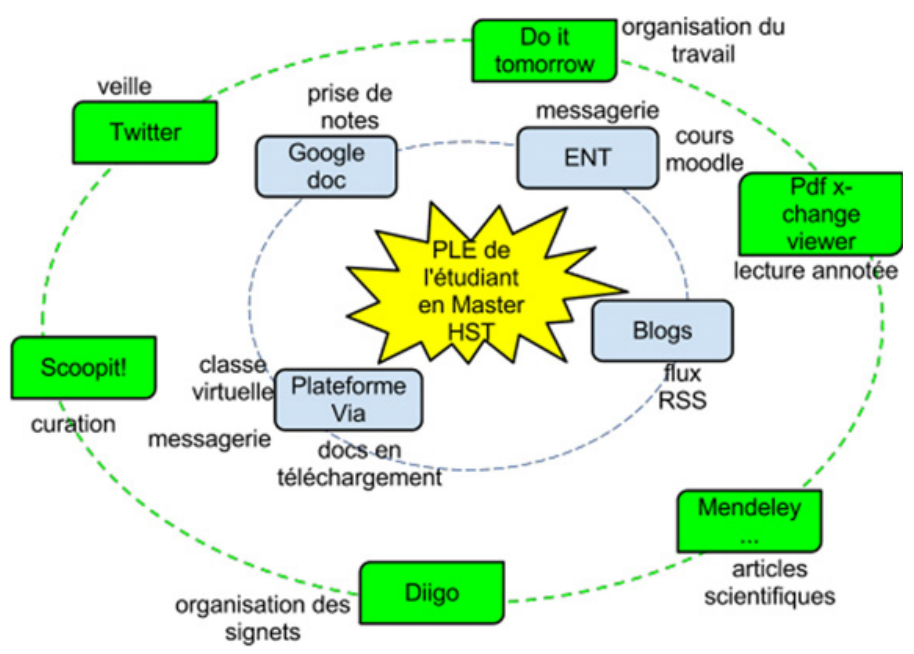

Par ailleurs, un blog (wordpress) est ouvert pour chaque étudiant. Sa fonction est multiple : apprendre et utiliser un outil de médiation et d'édition dans un usage récurrent (qui prépare à l'usage des carnets de recherche dans hypotheses.org), publier les résultats des différents travaux qui leur sont demandés, évaluer de la part des enseignants la production des étudiants en termes de médiation et de recherche ${ }^{33}$.

Comme nous l'avons déjà indiqué plus haut (\$4), nous favorisons l'interaction avec le milieu professionnel au travers de l'intervention d'un nombre conséquents d'intervenants issus de divers horizons en les questionnant sur leur rapport à la recherche en SHS, l'usage des TIC et la médiation

\footnotetext{
31 Voir https://wiki.univ-rennes1.fr/aide/doku.php?id=cirm:svi:svi_accueil (consulté 9.10.14).

32 Ce schéma a été produit par un de nos étudiants de master 2 en 2013.

33 A titre d'exemples, voir http://python.espe-bretagne.fr/blog-maeva-boudjema/?author=1; http://python . espe-bretagne.fr/blog-david-gomes/ (consulté 9.10.14).
} 
culturelle. Par ailleurs, Anne Rognant, responsable du service éducatif d'Océanopolis, prend en charge les enseignements concernant les médiations culturelles et les méthodes associées.

Nous cherchons aussi à inscrire la formation dans une pratique de l'anglais pour la recherche au travers d'une UE dédiée à la pratique de la langue orale et écrite par l'étude de conférences en HST en ligne, la production de fiches de lecture ainsi que l'intervention de collègues étrangers que nous invitons à parler en anglais.

Enfin, le choix et le travail des sujets de recherche s'effectuent avec la procédure suivante : des thématiques de recherche proposées par les enseignants du master et en connexion directe avec des opérations de recherche en cours, un temps important au premier semestre consacré à la construction d'une problématique assortie d'un repérage et d'une justification des sources qui seront étudiées, de la prise en compte de l'usage des TIC ainsi que des questions de médiation culturelles. Une fois validé, l'étudiant se consacre à la résolution de problème scientifique au cours du second semestre. Le mémoire final doit nécessairement comporter les trois aspects attendus (HST, TIC et médiation culturelle) avec la possibilité de donner une coloration forte dans un des trois domaines.

Pour terminer sur cette question de la mise en œuvre de l'enseignement, nous favorisons un enseignement basé sur la résolution de problèmes associées à des cours magistraux dans un croisement régulier des contenus et une approche pluridisciplinaire.

\section{Conclusion}

Ce master recherche a été pensé à partir de plusieurs prises de partie afin de développer des compétences et des savoirs leur permettant d'aborder dans de bonnes conditions une thèse portant sur les thématiques développées dans le master (HST, humanités numériques, médiations culturelles) ou de s'insérer dans les milieux professionnels en lien avec l'éducation, la médiation culturelle en sciences et techniques ou le développement d'outils muséologiques/pédagogiques innovants en lien avec la recherche. Il s'agit donc ici d'immerger les étudiants au plus près de la réalité de nos pratiques et de nos problématiques de recherche, de les initier à la gestion de projets pluridisciplinaires, de connaître les nombreux et divers terrains professionnels en jeu lorsqu'un projet de recherche comportant une part de valorisation de patrimoine se construit.

Enfin, la dimension recherche en "humanités numériques » nous parait absolument cruciale à aborder. Il s'agit d'un domaine en émergence qui pose question en termes de pratiques de recherche, d'épistémologie de l'HST comme des modes de communication des résultats de la recherche en HST. Le laboratoire comme le master s'inscrit résolument dans cette émergence qui est passionnante à tout point de vue, notamment dans le travail inter ou transdisciplinaire que cela implique avec les laboratoires STIC.

\section{Références}

[1] Bernard, A. 2009. « De l'intérêt de l'histoire des sciences dans l'enseignement secondaire et dans la formation des enseignants : état des lieux et réflexions ». Argos 45. http: //www . educrevues.fr/ARGOS/AffichageDocument . aspx?iddoc=38896 (consulté 9.10.14).

[2] Bernard, A. Chambon, G. Ehrhardt C. 2010, Le sens des nombres: mesures, valeurs et informations chiffrées : une approche historique, Vuibert/ ADAPT-SNES : Paris. http://www. adapt.snes.edu/spip.php?article617

[3] Bruneau, O, De Vittori, T, Grapi, P, Heering, P, Laubé, S, Massa-Esteve, M.R. 2012. Innovative Methods for Science Education: History of science, ICT and Inquiry Based Science Teaching, Frank \& Timme GmbH: Berlin. 
Les sciences humaines dans les parcours scientifiques et techniques professionnalisants

[4] Ferrière, H. 2011. L'homme, un singe comme les autres : éléments d'histoire et d'épistémologie pour enseigner l'évolution, Vuibert/ ADAPT-SNES : Paris.

[5] Gille, B. 1979. " La notion de système technique ». Cultures techniques, 1, 8-18. http://documents.irevues.inist.fr/handle/2042/30607

[6] Hughes, T. P. 1987. "The Evolution of Large Technological Systems". In Bijker, Hughes, \& Pinch (Eds.), The Social Construction of Technological Systems, pp. 51-82).

[7] Le Moigne, J.L. 2004. La Théorie du Système Général, Théorie de la Modélisation, PUF (rééditions complétées en 1983, 1990, 1994). Nouvelle édition complétée en 2004 : http: //www . intelligence-complexite.org/fr/bibliotheque/bibliotheque-du-ric/ouvrage/ la-theorie-du-systeme-general.html?tx_mcxapc_pi1 [action] =ouvrageDetail\& cHash=7f57246ec0fda3d4f83bd5613db99b84 (consulté 9.10.14).

[8] Lemonnier, P. 2010. «L'étude des systèmes techniques : une urgence en technologie culturelle », Cultures matérielles 54-55.

[9] Mauss M. 1967. Manuel d'ethnographie. Paris : Éditions sociales, Petite Bibliothèque Payot. http://classiques.uqac.ca/classiques/mauss_marcel/manuel_ethnographie/manuel_ ethnographie.html

[10] Rocher, Guy. 1992. «Culture, civilisation et idéologie ». In Introduction à la sociologie, Première partie: L'action sociale, chapitre 4, pp. 101-127. Montréal: Les Éditions Hurtubise HMH, 3e édition. Réimpression, 1995.

[11] Rosselin C. \& Julien M.-P.. 2005. La culture matérielle. La Découverte, coll. « Repères ».

\section{Travaux d'étudiants consultables en ligne}

[12] Boudjema, M. http://python.espe-bretagne.fr/blog-maeva-boudjema/?author=1

[13] Gomes, D. http://python.espe-bretagne.fr/blog-david-gomes

[14] Le Gall, C. projet LIBROS: http://liaison-lycees-ubo.univ-brest.fr/pages/libros

[15] Le Gall C. http://poleni.hypotheses.org/

[16] Rohou, B.http://brmdp.hypotheses.org/ 